\title{
Linfoma difuso de células B grandes gástrico primario
}

\section{Primary gastric diffuse large B-cell lymphoma}

\author{
José Gonzalo Huamán-Muñante', \\ José Luis Salinas-Paz', \\ Luis Ricardo Arangüena-Necochea', \\ José Ramiro Cachay-Díaz', \\ Magaly Silvia Carpio-Laucata ${ }^{3}$
}

Huamán-Muñante JG, Salinas-Paz JL, Aranguena-Necochea LR, CachayDíaz JR, Carpio-Laucata MS. Linfoma difuso de células B grandes gástrico primario. $2021 ; 34(2): 76-81$. https://doi.org/10.36393/spmi.v34i2.601

\begin{abstract}
RESUMEN
Paciente mujer de 37 años, quien ingresa en mal estado general con alteración de la conciencia y derrame pleural. Cinco meses antes de su ingreso presenta dolor epigástrico intenso y recurrente, dispepsia, pérdida de peso y sudoración nocturna. Fue diagnosticada de gastritis y le prescribieron sintomáticos con leve mejoría. Pero los síntomas persistieron. Los estudios de imágenes e histopatológicos y de inmunohistoquímica llevaron al diagnóstico de linfoma no-Hodgkin de células grandes B difuso fenotipo centro germinal primario gástrico. La paciente fue con ciclofosfamida, doxorrubicina, vincristina y prednisona, más rituximab. La evolución fue favorable.
\end{abstract}

Palabras claves: linfoma no-Hodgkin, cáncer gástrico, rituximab.

\section{ABSTRACT}

A 37-year-old female patient who was admitted in poor general condition with altered consciousness and pleural effusion. Five months before admission, she had severe and recurrent epigastric pain, dyspepsia, weight loss and night sweats. She was diagnosed with gastritis and prescribed with relief drugs A slight improvement and the symptoms persisted. Imaging, histopathological and immunohistochemistry studies led to

Médico internista. Servicio de Medicina Interna, Hospital Nacional Arzobispo Loayza, Lima, Perú

2 Médico oncólogo. Servicio de Oncohematología, Hospital Nacional Arzobispo Loayza, Lima, Perú

3 Estudiante de medicina, Servicio de Medicina Interna, Hospital Nacional Arzobispo Loayza, Lima, Perú the diagnosis of diffuse large cell B non-Hodgkin lymphoma gastric primary germinal center phenotype. The patient was on cyclophosphamide, doxorubicin, vincristine and prednisone, plus rituximab. The evolution was favorable.

Keywords: non-Hodgkin lymphoma, gastric cancer, rituximab.

\section{INTRODUCCIÓN}

El adenocarcinoma, el proceso maligno más frecuente del estómago, representa más del $90 \%$ de todos los cánceres gástricos. ${ }^{1}$ Los linfomas gástricos son de menor frecuencia que los adenocarcinomas y con mayor tasa de curación.

Los linfomas son tumores sólidos del sistema linfático, que se subdividen en linfomas Hodgkin y no-Hodgkin (LNH), éstos últimos, constituyen a su vez, un grupo heterogéneo de neoplasias y pueden originarse en tejidos no ganglionares como el tubo digestivo. ${ }^{2,3}$ De modo particular, los linfomas 
gastrointestinales, entre ellos, los linfomas gástricos, que son neoplasias malignas hematológicas de origen extranodal, constituyen el principal sitio de origen de los linfomas no ganglionares, pese a su baja incidencia dentro de los tumores gástricos malignos, donde se constituyen entre el 2 y el 5\% de ellos. ${ }^{4-5}$

Dentro de los LNH, el linfoma difuso de linfocitos B grandes (LDLBG) es su forma más frecuente y constituyen algo más del 60\% de ellos. Cada año, en EE.UU. se presentan 25000 casos nuevos, con un ligero predominio en los varones, siendo 60 años la edad promedio de los pacientes con dicha afección; no obstante, el LDLBG también se presenta en adultos jóvenes y niños. ${ }^{1}$

Se desconoce la causa exacta del $\mathrm{LNH}$, aunque algunas evidencias sugieren una causa viral y también otros factores ambientales que desencadenan alteraciones genéticas y/o epigenéticas, pero se conocen dos factores de riesgo importantes: infección y desregulación inmunológica. Un ejemplo claro de ello es la asociación del Helicobacter pilory y el linfoma asociado a los tejidos de la mucosa gástrica (MALT).

Se presenta el caso de una paciente con linfoma no-Hodgkin de células grandes b difuso fenotipo centro germinal primario gástrico.

\section{PRESENTACIÓN DEL CASO}

Paciente de sexo femenino, de 37 años de edad, ama de casa, natural de Loreto, que ingresó al servicio de emergencia en mal estado general, refiriendo un tiempo de enfermedad de unos cinco meses antes de su hospitalización, con dolor abdominal que se incrementa de manera progresiva en intensidad y frecuencia, se asocia a náuseas, vómitos inicialmente de contenido blanquecino y bilioso y luego porráceos; además, distensión abdominal, saciedad precoz, pesadez, indigestión, eructos mal olientes. Acudió a consulta médica en una clínica privada donde le diagnosticaron gastritis y le prescribieron inhibidores de bomba de protones y analgésicos, con mejoría leve y transitoria; sin embargo, los síntomas persistieron y se agravaron en los meses siguientes. Se añadió un olor fétido en la región umbilical y cambios en la piel, astenia, disminución de la diuresis, dolor de tobillos, dificultad respiratoria progresiva y fiebre de $39^{\circ} \mathrm{C}$, y sudoración nocturna, por los que acudió al hospital.

Funciones biológicas: apetito disminuido; sed disminuida; orina disminuida; deposiciones diarreicas intermitentes; sueño interrumpido y con sudor nocturno; peso disminuido en $17 \mathrm{~kg}$ durante los últimos tres meses.

Antecedentes patológicos: Solo refería un dolor abdominal recurrente desde hace varios años.

Examen Físico: mal estado general, alteración de la conciencia, con facies dolorosa, disneica, asténica, pálida. PA $115 / 80 \mathrm{~mm} \mathrm{Hg}$; temperatura $36^{\circ} \mathrm{C}$, frecuencia cardiaca 115 latidos/min, frecuencia respiratoria 28 excursiones/ min. Talla 1,49 metro; peso $58 \mathrm{~kg}$, IMC 26,3.

Piel con palidez moderada, edema $1+/ 4+$ en los miembros inferiores. Sistema linfático: presencia de adenopatías ganglionares, inguinal, axilar y cervical. SOMA: actividad muscular y articular normal.

Respiratorio: asimetría torácica izquierda, matidez y murmullo vesicular disminuido en la base izquierda. Cardiovascular: ruidos cardiacos normales, no ruidos agregados. Abdomen: distendido, globuloso, ruidos hidroaéreos incrementados; blando, depresible; doloroso a la palpación en el cuadrante superior izquierdo, impresiona palparse masa poco definible en el epigastrio, no se palpa bazo, hígado en el reborde costal derecho, no impresiona ascitis. Neurológico: Glasgow 13/15, no focalización, fuerza muscular conservada.

Estudios de Laboratorio

- Leucocitos 16 500/mm³ $\mathrm{mb}^{3}$ 8,4 g/dl, VCM80 fl, HBCM $24 \mathrm{pg} /$, microcitosis 1+, hipocromía 3+, anisocitosis 1+; plaquetas $280000 / \mathrm{mm}^{3}$.

Glucosa $65 \mathrm{mg} / \mathrm{dl}$, creatinina $0,25 \mathrm{mg} / \mathrm{dl}$, albúmina 2,36 $\mathrm{g} / \mathrm{dl}$, globulinas $3,17 \mathrm{~g} / \mathrm{dl}$, bilirrubina total $0,30 \mathrm{mg} / \mathrm{dl}$, bilirrubina directa $0,15 \mathrm{mg} / \mathrm{dl}$, ácido úrico $2,4 \mathrm{mg} / \mathrm{dl}$ (VN $2.37 \mathrm{mg} / \mathrm{dl}$ ), TGO $18 \mathrm{U} / \mathrm{L}$, TGP $8 \mathrm{U} / \mathrm{L}$, fosfatasa alcalina 58 U/L, GGT 11 U/L, amilasa 36 U/L, lipasa 9 U/L, DHL 1403 (VN 50-450), calcio sérico 8,4mg/dl ( $\mathrm{VN} \mathrm{8,6-10,2} \mathrm{mg/dl),}$ proteína $\mathrm{C}$ reactiva $2,5 \mathrm{mg} / \mathrm{dl}$ ( $\mathrm{VN} 0-0,5)$, AgHBs negativo, VDRL no reactivo, VIH anticuerpos no reactivo, HTLV I y II no reactivo, beta-2 microglobulina 3,8 mg/dl (VN 0,8-2,2 $\mathrm{mg} / \mathrm{dl}$ ), Ca 72-4 (estómago) 1,12 U/ml (VN hasta 6,9 U/ $\mathrm{ml})$, Ca 19-9 0,88 U/ml (VN 0-39 U/ml), CEA 1,62 ng/ml (VN 0-5 ng/ml).

Se realizó una toracocentesis. Líquido pleural: recuento. Celular 2000 células/mm3 (PMN 90\%); glucosa 90mg/d, proteínas 2,68mg/dl, DHL 1467 U/L, ADA: 17 U/L (VN:0$30 \mathrm{U} / \mathrm{L}$, ). Gram: no se encuentran gérmenes; cultivo negativo.

En la radiografía de tórax: presencia de efusión pleural masiva izquierda, con desplazamiento contralateral de mediastino, borramiento de la silueta diafragmática y obturación de senos costo-frénico izquierdo y cardiofrénico izquierdo (Figura 1).

Ecografía abdominal: se observa un engrosamiento irregular de la pared gástrica, por lo cual se sugiere endoscopia (Figura 2).

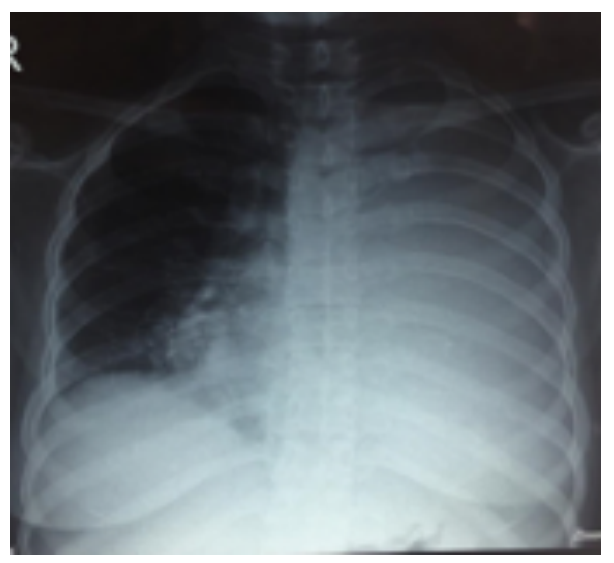

Figura I. Radiografía de tórax: derrame pleural izquierdo. 


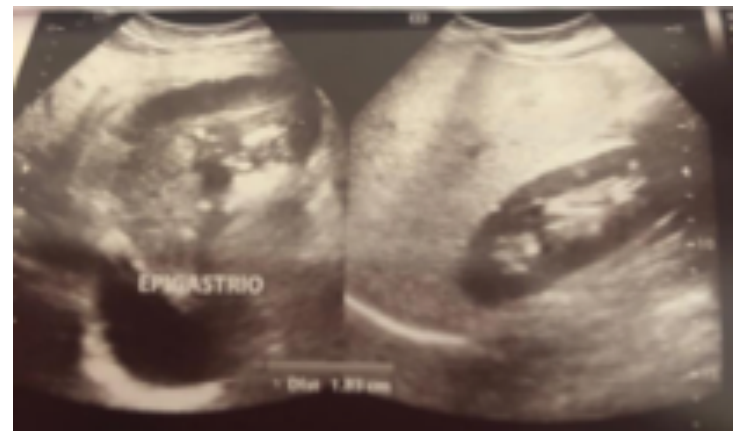

Figura 2. Ecografía: Se observa un engrosamiento irregular de la pared gástrica.

En la tomografía de abdomen: engrosamiento mural difuso en estomago de aspecto neoformativo con infiltración local y adenopatías perigástricas, tronco celiaco y retroperitoneo, con ascitis leve (Figura 3).

TEM de Tórax: gran efusión pleural izquierda con engrosamiento pleural y atelectasia lobar inferior izquierda. En la endoscopía digestiva alta: presencia de lesión amplia de aspecto infiltrativo con áreas ulceradas que compromete región subcardial, fondo gástrico y cuerpo proximal y medio hacia curvatura mayor y menor hacia ángulo, con áreas de fibrina y friabilidad al roce. Dicha lesión compromete la distensibilidad gástrica y se evidencia retención de alimentos, clasificada como una neoplasia maligna gástrica, Bormann III.
Anatomopatología: en el estudio de la biopsia gástrica, se observa abundante tejido infiltrado de células B de tipo difuso, linfocitos neoplásicos grandes, abundante mitosis, pleomorfismo nuclear y atipia celular (Figuras. 5-7).

Inmunohistoquímica: alta expresión del CD 20 en las membranas de células neoplásicas (Figura 8). El índice proliferativo es de $60 \%$ en la muestra Ki67 (Figura 9). La mayoría de las células marca BCL-2 + (Figura 10), el cual desempeña un rol importante en la inhibición de la apoptosis (en algunos tipos de cánceres se relaciona con mejor pronóstico, mama y pulmón; sin embargo, en los cánceres gástricos está aún en investigación).

En la figura 11, marca en la mayoría C-MYC + que participa en la regulación del ciclo celular. "Dichos genes actúan como factores de transcripción y reguladores del ciclo celular e intervienen en la proliferación, apoptosis y diferenciación celular y en la inmortalización.” ${ }^{6}$ En las neoplasias con defectos de C-MYC los estudios están dirigidos al desarrollo e investigación de nuevas estrategias terapéuticas.

No se identificó presencia de Helicobacter pylori en el tejido biopsiado, pues no se tuvo muestra del tejido normal.

El examen físico, los exámenes auxiliares y el estudio anatomo patológico e inmunohistoquímico, éste último como el gold standard para el diagnóstico, fueron necesarios para llegar al diagnóstico definitivo de linfoma no Hodgkin de células grandes $\mathrm{B}$ difuso fenotipo centro germinal primario gástrico, neoplasia que es de mejor pronóstico que el adenocarcinoma gástrico.
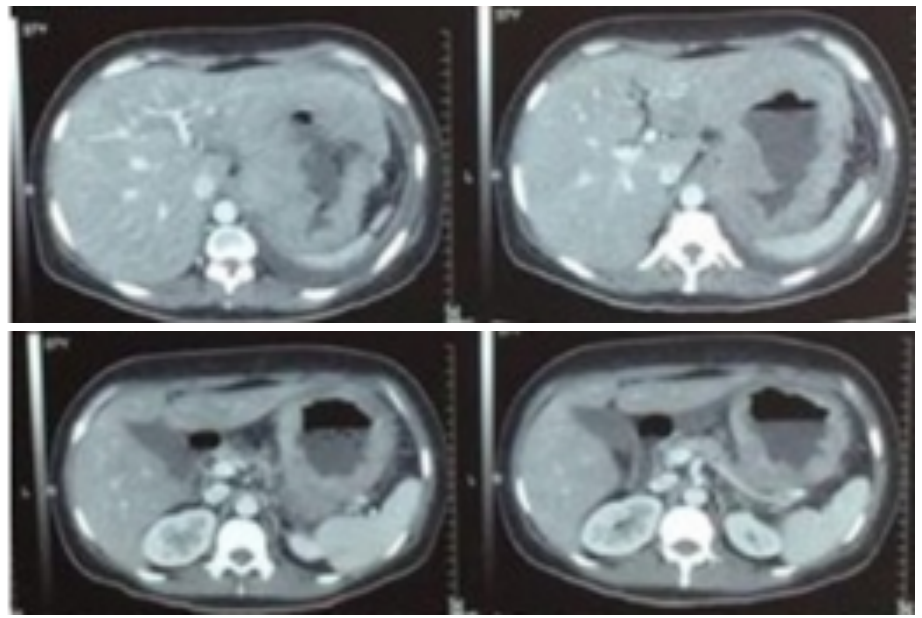

Figura 3. TAC abdominal: engrosamiento de pared gástrica.

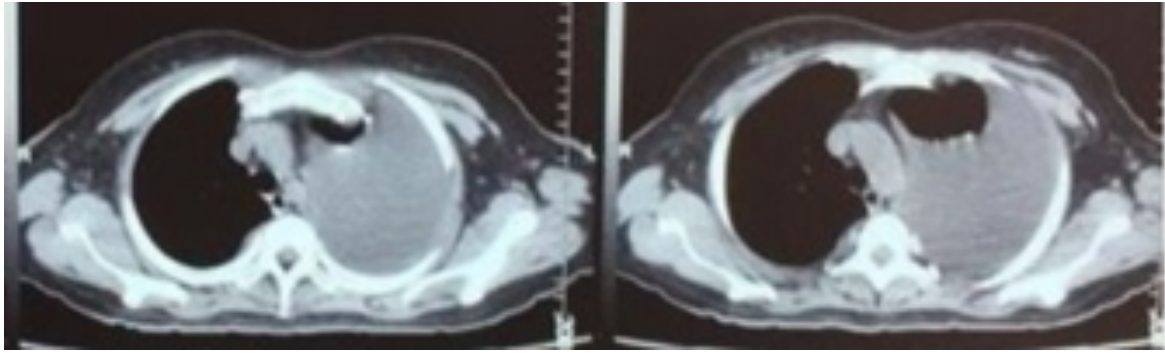

Figura 4. TEM de tórax, efusión pleural izquierda masiva. 


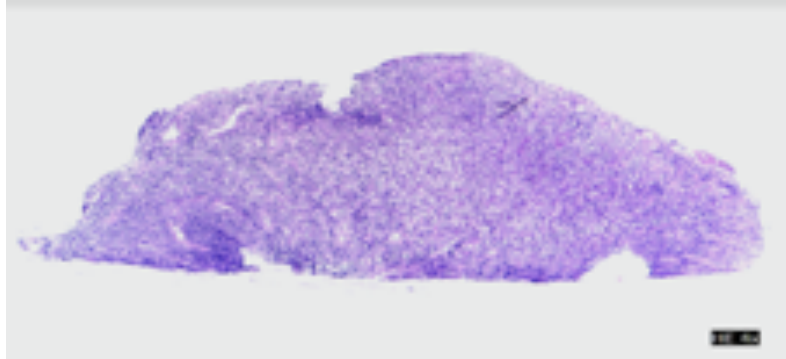

Figura 5. Corte histopatológico de Linfoma no-Hodgkin 4x.

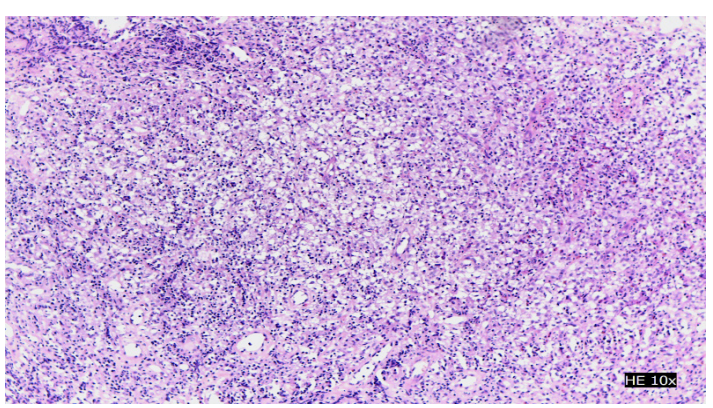

Figura 6. Tejido infiltrado de células B de tipo difuso, linfocitos neoplásicos grandes, abundante mitosis, pleomorfismo nuclear, atipia celular

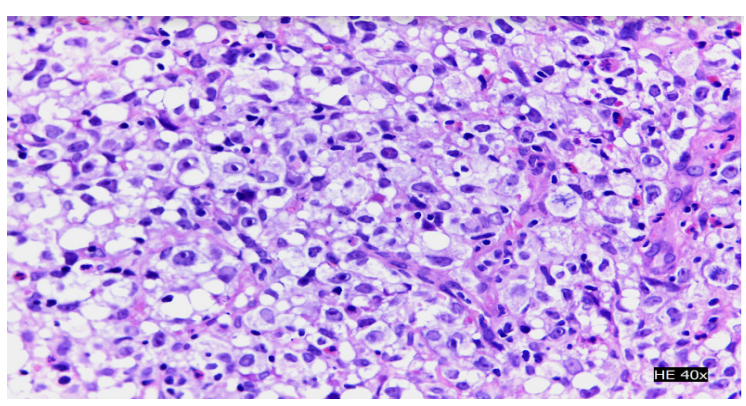

Figura 7. Conglomerado de células neoplásicas, presencia de linfocitos B grandes, difuso tipo centro germinal.

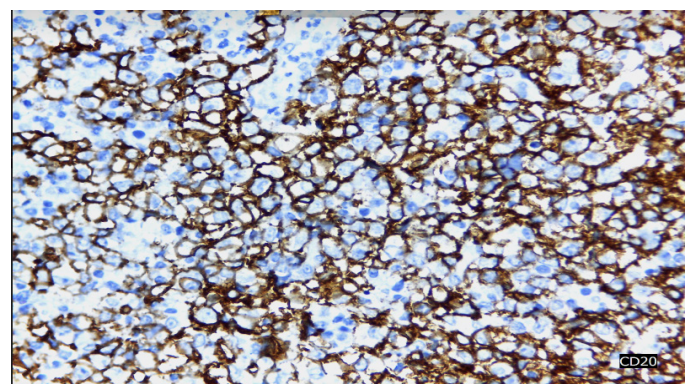

Figura 8. Inmunohistoquímica, en la mayoría marca CD20 +.

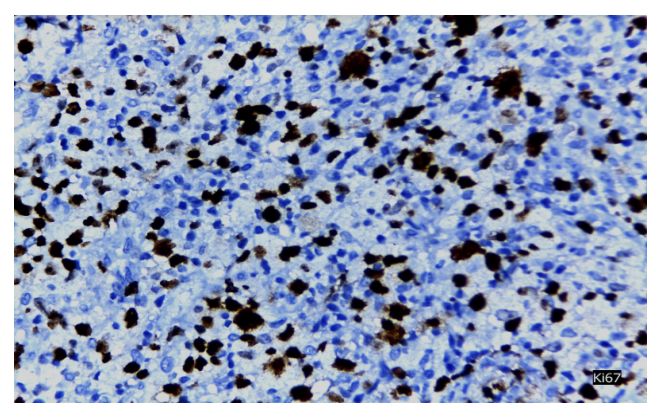

Figura 9. Inmunohistoquímica, marca el índice proliferativo de $60 \% \mathrm{Ki} 67$.

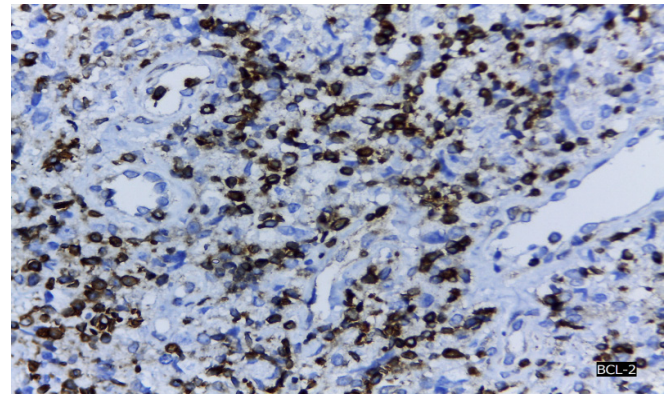

Figura I0. Inmunohistoquímica, en la mayoría marca BCL-2 +

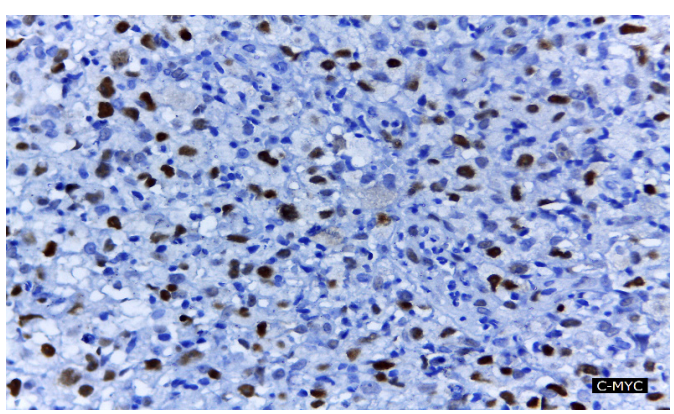

Figura II. Inmunohistoquímica, en la mayoría marca C-MYC +.

Debido a la infiltración gastro intestinal extensa y a los múltiples sitios de afectación extra ganglionares, tanto infra como supra diafragmáticos, le correspondió un estadio IV de acuerdo con clasificación modificada de los Estadios Ann-Arbor (Cuadro 1).

\begin{tabular}{|c|l|}
\hline \multicolumn{2}{|c|}{$\begin{array}{l}\text { Cuadro 1. } \\
\text { Clasificación de Ann Arbor modificado para el estadiaje de LNH. }\end{array}$} \\
\hline I & $\begin{array}{l}\text { Afecta a una sola región ganglionar (I) o se localiza en un solo órgano } \\
\text { o área extralinfática (IE). }\end{array}$ \\
\hline II & $\begin{array}{l}\text { Afecta a dos o más regiones ganglionares del mismo lado del } \\
\text { diafragma (II) o una afectación extralinfática y en una o más regiones } \\
\text { de ganglios linfáticos del mismo lado del diafragma (IIE) }\end{array}$ \\
\hline III & $\begin{array}{l}\text { Afecta a dos o más regiones ganglionares en ambos lados del } \\
\text { diafragma (III), que también puede estar acompañada de afectación } \\
\text { extralinfática localizada (IIIE) o de afectación esplénica (IIIS). }\end{array}$ \\
\hline IV & $\begin{array}{l}\text { Afecta de forma difusa o diseminada a uno o más órganos o tejidos } \\
\text { extralinfáticos, asociada o no con afectación de ganglios linfáticos. La } \\
\text { afectación de la medula ósea o hepática siempre se considerará como } \\
\text { estadio IV. }\end{array}$ \\
\hline
\end{tabular}

Para establecer el diagnóstico de linfoma primario gástrico es imprescindible diferenciarlo de un linfoma sistémico con afectación secundaria del estómago. En este caso el cuadro clínico y la lesión predominante presente en estómago en las imágenes dan sustento a la afectación primaria de estómago. Los criterios de Dawson aplicados al presente caso refuerzan dicha postura (Cuadro 2).

El linfoma no Hodgkin, de linaje B, CD 20+ es tributario al tratamiento con rituximab, un anticuerpo monoclonal dirigido contra el CD 20, el cual se inicia de manera inmediata. Como lo dicta el estadiaje del presente caso, para el abordaje terapéutico inicial, el paciente recibió el esquema de quimioterapia combinada de R-CHOP. 


\begin{tabular}{|l|}
\hline \multicolumn{1}{c|}{$\begin{array}{c}\text { Cuadro 2. } \\
\text { Criterios de Dawson. }{ }^{12}\end{array}$} \\
\hline Ausencia de linfadenopatías periféricas. \\
\hline Ausencia de linfadenopatías mediastinales en la radiografia de tórax. \\
\hline Frotis de sangre periférica normal. \\
\hline $\begin{array}{l}\text { Durante la laparotomía el compromiso de sólo esófago, estómago y el } \\
\text { intestino o de sólo ganglios regionales. }\end{array}$ \\
\hline $\begin{array}{l}\text { Ausencia de compromiso hepático y esplénico; excepto por la diseminación } \\
\text { directa de la enfermedad a partir de un foco contiguo }\end{array}$ \\
\hline
\end{tabular}

Luego de efectuar el tratamiento R-CHOP anti CD 20 a la paciente por aproximadamente tres meses, se observa en la Figura 12 una radiografía normal donde no hay presencia de masa tumoral.

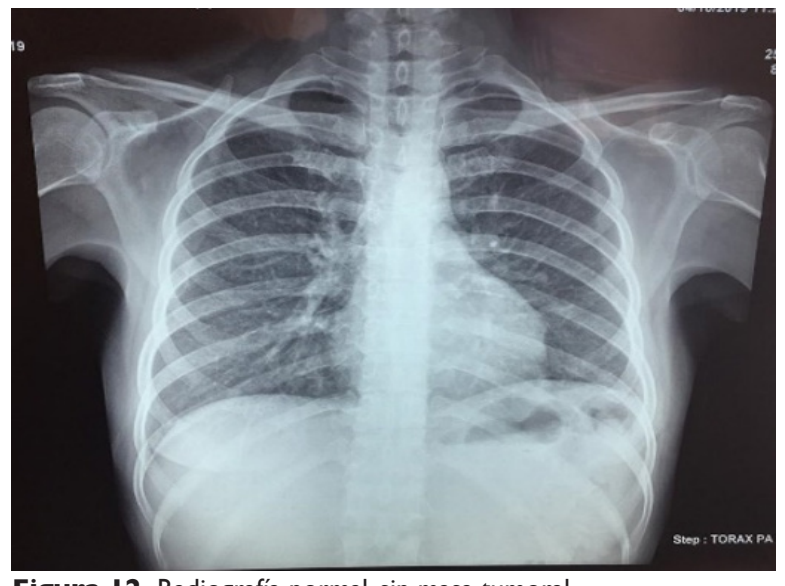

Figura I2. Radiografía normal, sin masa tumoral.

Para determinar los factores pronósticos que permitieran predecir la probable evolución clínica de la paciente se aplicó el Índice Pronóstico Internacional (IPI) de linfomas no-Hodgkin agresivo, dando el valor intermedio alto. En esta evaluación se toman los siguientes parámetros: edad, estado general, valor de la deshidrogenasa láctica (DHL), estadio y la presencia de toma extra ganglionar.

\section{DISCUSIÓN}

En el análisis inicial del presente caso, se identificaron varios problemas clínicos que requerían un adecuado abordaje. En primer lugar, el dolor abdominal crónico persistente y progresivo, el cual nos indica organicidad y asociado a baja ponderal, anemia, intolerancia oral, masa palpable en el epigastrio o hemorragia digestiva que hizo pertinente y necesaria un estudio endoscópico. En segundo lugar, los hallazgos de anemia ferropénica y signos de compromiso extraabdominal inexistente al principio, pero evidente en la evolución. ${ }^{7-9}$ Lo primero suele ser explicado por sangrado crónico del tubo digestivo como lo sugiere los vómitos borráceos del paciente; y, por compromiso metastásico. El compromiso pleural presente en la evolución tardía de los síntomas nos sugirió secundarismo y que la afectación gástrica fue la afectación primaria. En tercer lugar, los estudios realizados como la endoscopia digestiva alta que resultó concluyente, más los estudios de imágenes que determinaron el estadiaje y el estudio histopatológico de la biopsia gástrica fueron determinantes para confirmar la sospecha clínica.

Mención aparte necesitan los marcadores oncológicos como el DHL y beta 2 microglobulina elevados, los cuales son también, aunque no definitivos, indicadores de neoplasia linfomatosa.

Los dolores abdominales leves e intermitentes no deben ser subestimados y más si estos son por tiempo prolongado, estos deben ser estudiados oportunamente para descartar cualquier neoplasia incipiente. Los hallazgos tempranos en las primeras etapas de las neoplasias son $100 \%$ curables. Cualquier gastritis diagnosticada que no responde al tratamiento con sintomáticos deben ser estudiados con una endoscopía alta y biopsia para determinar la etiología exacta de la enfermedad y dar el tratamiento oportuno. Los síntomas B (fiebre, pérdida de peso y sudoración nocturna) son característicos de los linfomas Hodgkin y no-Hodgkin. En este tipo de neoplasias malignas la necesidad de realizar una cirugía no está indicada sobre todo en individuos con signos radiográficos preoperatorios con metástasis ganglionares, en quienes la quimioterapia por si sola como R-CHOP (ciclofosfamida, doxorrubicina, vincristina $y$ prednisona) más rituximab constituye un tratamiento eficaz y se recomienda como tratamiento de primera elección incluso, en los estadios clínicos III-IV, grado, de evidencia fuerte a favor en todas las guías internacionales (Categoría 1, NCCN; nivel de evidencia I, ESMO; nivel de evidencia 1A, BCSH/BHS)..$^{10}$ La realización de la biopsia, en este caso particular, debe ampliarse también a tejido sano, a fin de documentar la infección por Helicobacter pylori, a menudo, involucrado en linfoma tipo MALT.

La presentación clínica habitual del LNHCGDB es la adenopatía cervical baja izquierda, bilateral, supraclavicular o supraaórtica sin compromiso mediastinal y los síntomas tipo B presentes, aunque no en la frecuencia de los LH. La afectación extraganglionar y de órganos internos suele ser diagnosticada de manera accidental pero se puede sospechar cuando los marcadores DHL y beta- 2 microglobulina se encuentran elevados en el contexto de sospecha de NM gástrico.

En conclusión, para el diagnóstico definitivo de linfoma no-Hodgkin primario gástrico de células grandes de linaje $\mathrm{B}$ centro germinal debe realizarse los estudios necesarios porque tienen mejor pronóstico.

\section{REFERENCIAS BIBLIOGRÁFICAS}

I. Kumar, V., A. K. Abbas, N. Fausto y J. C. Aster. Robbins y Cotran Patología estructural y funcional. Ed. Elsevier, $9^{a}$ ed. 20I 5: 596, 77I.

2. Olszewski AJ, Castillo J. Survival of patients with marginal zone lymphoma: analysis of the surveillance, epidemiology, and end results database. Cancer. 2013;119(3):629-38.

3. Bautista-Quach MA, Ake CD, Chen M, Wang J. Gastrointestinal lymphomas: morphology, immunophenotype and molecular features. J Gastrointest Oncol. 2012;3(3):209-25.

4. Pisano R, Llorens P, Durán V,Altschiller G, Goldin L, Burmeister R, et al: Linfomas gástricos primarios: aspectos clínicos e histológicos en 
4 I casos. Rev Méd Chile. 1988; | 16: I27|-6.

5. López F, Llanos O, Guzmán S, López C, Duarte I, Lezana G: Linfoma no Hodgkin del estómago: evaluación del tratamiento quirúrgico en 24 pacientes. Rev Méd Chile 1994; I 22: 1378-84.

6. Gustafson WC, Weiss WA. Myc proteins as therapeutic targets. Oncogene. 2010 Mar 4;29(9):1249-59.

7. Costa R. et al. Linfoma não Hodgkin gástrico. Revista Brasileira de Hematologia e Hemoterapia. 2010, 32(I): 63-69.

8. Beltran Gárate B, Morales Luna D, Quiñones Avila P, Hurtado De Mendoza F, Riva Gonzales L, et al. linfoma de células grandes B difuso primario colorrectal: Experiencia en un Hospital General. Revista Gastroenterología Perú. 2007; 28: 235-238.
9. Butte J, Torres J, Duarte I, Guzmán S, Llanos O. Adenocarcinoma gástrico treinta y dos años post linfoma gástrico. Revista médica de Chile. 2008; |36(I0): I3 |7-1320.

10. Comité de Guías de Práctica Clínica Red AUNA. Guía de Práctica Clínica, Manejo Multidisciplinario del Linfoma no Hodgkin de Célula B Grandes Difuso,AUNA, Lima: 2019.

\section{CORRESPONDENCIA:}

Magaly Silvia Carpio-Laucata,

maggyta@gmail.com

Fecha de recepción: |6-04-202|

Fecha de aceptación:

Financiamiento: por los autores.

Conflicto de interés: ninguno, según los autores. 